\title{
Diego Orlando
}

Dinâmica Não-Linear, Instabilidade e Controle de Sistemas Estruturais com Interação Modal

Tese apresentada como requisito parcial para obtenção do título de Doutor pelo Programa de PósGraduação em Engenharia Civil da PUC-Rio. Área de Concentração: Estruturas.

Orientadores: Paulo Batista Gonçalves

Giuseppe Rega

Stefano Lenci 
Diego Orlando

\title{
Dinâmica Não-Linear, Instabilidade e Controle de Sistemas Estruturais com Interação Modal
}

\begin{abstract}
Tese apresentada como requisito parcial para obtenção do título de Doutor pelo Programa de Pós-Graduação em Engenharia Civil da PUC-Rio. Aprovada pela Comissão Examinadora abaixo assinada.
\end{abstract}

\author{
Prof. Paulo Batista Gonçalves \\ Presidente/Orientador \\ Departamento de Engenharia Civil - PUC-Rio \\ Prof. Carlos Eduardo Nigro Mazzilli \\ USP - SP \\ Prof. Marcelo Amorim Savi \\ COPPE/UFRJ \\ Prof. Raul Rosas e Silva \\ PUC-Rio \\ Prof. Deane de Mesquita Roehl \\ PUC-Rio \\ Prof. José Eugênio Leal
}

Coordenador(a) Setorial do Centro Técnico Científico - PUC-Rio

Rio de Janeiro, 07 de maio de 2010 
Todos os direitos reservados. É proibida a reprodução total ou parcial do trabalho sem autorização da universidade, do autor e do orientador.

\section{Diego Orlando}

Graduou-se em Engenharia Civil pela Universidade de Passo Fundo (UPF), em janeiro de 2004. Ingressou no mestrado em Engenharia Civil da PUC-Rio em março de 2004, atuando na área de Instabilidade e Dinâmica das Estruturas. Em 2006, continuando na mesma linha de pesquisa, iniciou o curso de doutorado na PUC-Rio.

Ficha Catalográfica

Orlando, Diego

Dinâmica não-linear, instabilidade e controle de sistemas estruturais com interação modal / Diego Orlando ; orientadores: Paulo Batista Gonçalves, Giuseppe Rega, Stefano Lenci. - 2010. $300 \mathrm{f}$. : il. (color.); $30 \mathrm{~cm}$

Tese (Doutorado)-Pontifícia Universidade Católica do Rio de Janeiro, Departamento de Engenharia Civil, Rio de Janeiro, 2010.

Inclui bibliografia

1. Engenharia civil - Teses. 2. Acoplamento modal. 3. Sensibilidade a imperfeições. 4. Integridade dinâmica. 5. Modos não-lineares. I. Gonçalves, Paulo Batista. II. Rega, Giuseppe. III. Lenci, Stefano. IV. Pontifícia Universidade Católica do Rio de Janeiro. Departamento de Engenharia Civil. V. Título. 
Dedico este trabalho como minha mais saudosa homenagem aos meus pais, Wilson Orlando e Melânia Maria Orlando, por todo amor e carinho. Ao meu irmão Thiago Orlando, pelo amor e amizade. 


\section{Agradecimentos}

Agradeço a vida, e àqueles que passam fazendo-a valer à pena.

Ao professor Paulo Batista Gonçalves pelas conversas, pelo constante auxílio, pela paciência e por sua amizade.

Aos professores Giuseppe Rega e Stefano Lenci, pessoas sensacionais, com qual tive o prazer de conviver e aprender muito.

Aos professores que participaram da comissão examinadora.

As pessoas que me estenderam as mãos quando mais precisei no período que passei na Itália, Irmãs Adelaide e Adriana obrigado.

Juntamente uni-se a Família Thompson, que não somente me estenderam as mãos, mas também abriram sua casa. Jack, Rosa, Olivia e Hannah, hoje, vocês fazem parte de minha família.

Aos grandes amigos Henrique Marek, Eduardo Mattos, Erblai Mattos Junior, Cleiton Batista Silvério, André Guimarães e Osmar Cervieri, que mesmo longe sempre me incentivaram e apoiaram.

Aos amigos Patrício e Juliana Pires, Walter Menezes, Thiago Pecin, André Müller, Frederico e Renata Alves, Eduardo Pasquetti, Magnus Meira, Joabson Alves, Julio e Gisele Holtz, Patrícia Cunha, Fernando Ramires e Alexandre Del Savio obrigado pelo incentivo e apoio.

Aos amigos e companheiros da sala 609, em especial José Silvestre, João Pantoja, Christiano Teixera, João Krause, Paul Antezana e Jean Aguilera.

Aos professores, engenheiros e amigos Zacarias Chamberlain e Gilnei Artur Drehmer pelo constante apoio e incentivo.

Aos demais professores do departamento de Engenharia Civil da PUC-Rio.

A Cnpq e a Capes pelo apoio financeiro, sem os quais este trabalho não poderia ser realizado.

Por fim, a todos aqueles que contribuíram na realização desta Tese. 


\section{Resumo}

Orlando, Diego; Gonçalves, Paulo Batista; Rega, Giuseppe; Lenci, Stefano. Dinâmica Não-Linear, Instabilidade e Controle de Sistemas Estruturais com Interação Modal. Rio de Janeiro, 2010. 300p. Tese de Doutorado Departamento de Engenharia Civil, Pontifícia Universidade Católica do Rio de Janeiro.

O objetivo desta tese de doutorado é estudar a influência do acoplamento de modos de flambagem no comportamento estático e particularmente no comportamento dinâmico não-linear de elementos estruturais suscetíveis a flambagem. Para isto, usam-se dois modelos discretos conhecidos por seu complexo comportamento não-linear: o modelo de Augusti e um modelo de torre estaiada com dois graus de liberdade. Inicialmente estuda-se a estabilidade dos dois modelos perfeitos, incluindo a obtenção de todos os caminhos de equilíbrio pré- e pós-críticos e o efeito das imperfeições na capacidade de carga da estrutura e na estabilidade dos diversos caminhos de equilíbrio. O objetivo desta análise é entender como as diversas soluções pós-críticas instáveis e as imperfeições influenciam a geometria da superfície de energia potencial, o contorno do vale potencial pré-crítico e a integridade da estrutura frente a inevitáveis perturbações externas. A seguir estuda-se o comportamento dos modelos em vibração livre. Após a identificação das freqüências naturais, dos modos lineares de vibração e das ressonâncias internas, estuda-se, com o objetivo de entender a dinâmica dos modelos, usando as ferramentas da mecânica Hamiltoniana, a geometria da região segura que circunda a posição de equilíbrio pré-crítica, cuja estabilidade se deseja preservar, e as variedades invariantes dos pontos de sela que definem esta região. Ainda, no contexto da análise das vibrações livres, determinam-se todos os modos não-lineares de vibração, sua estabilidade e sua relação freqüência-amplitude. Estes modos não-lineares estáveis e instáveis, que surgem em virtude do acoplamento modal e das simetrias dos modelos, controlam e explicam a sua dinâmica sob vibração forçada. Com base nesses resultados, estuda-se o comportamento dos modelos sob uma excitação de base, através de um estudo sistemático de bifurcações globais e locais, e a integridade das soluções estáveis através da evolução e estratificação das bacias de atração e das medidas de integridade dinâmica. Finalmente estuda-se como aumentar a segurança da 
estrutura através do controle das bifurcações globais homoclínicas e heteroclínicas. A presente tese revela um conjunto de comportamentos que são típicos dos dois modelos e que podem ser entendidos como fenômenos característicos de estruturas que exibem acoplamento modal. Assim, a principal contribuição deste trabalho reside na identificação de algumas características e aspectos particulares dessa classe de estruturas, assunto inédito na literatura.

\section{Palavras-chave}

Acoplamento modal, sensibilidade a imperfeições, integridade dinâmica e modos não-lineares. 


\section{Abstract}

Orlando, Diego; Gonçalves, Paulo Batista; Rega, Giuseppe; Lenci, Stefano. Nonlinear Dynamics, Instability and Control of Structural Systems with Modal Interaction. Rio de Janeiro, 2010. 300p. Tese de Doutorado Departamento de Engenharia Civil, Pontifícia Universidade Católica do Rio de Janeiro.

The aim of this thesis is to study the influence of coupled buckling modes on the static and particularly on the nonlinear dynamic behavior of structural components liable to buckling. For this, two discrete two degrees of freedom models known for their complex nonlinear behavior are selected: the well-known Augusti's model and a simplified model of cable-stayed tower. Initially, the stability analysis of the perfect models is conducted, including the identification of all pre- and post-critical equilibrium paths, and the effect of imperfections on the load capacity of the structure and stability of the various equilibrium paths. The purpose of this analysis is to understand how the various unstable post-critical solutions and imperfections influence the geometry of the potential energy surface, the contour of the pre-buckling potential well and the integrity of the structure under the inevitable external disturbances. Then the behavior of the models in free vibration is investigated, including the identification of the natural frequencies, linear vibration modes and possible internal resonance. To understand the dynamics of the models, the geometry of the safe region surrounding the pre-buckling equilibrium position and the invariant manifolds of saddle points that define this region are obtained using the tools of Hamiltonian mechanics. Also, as part of the free vibrations analysis, all stable and unstable nonlinear vibration modes and their frequency-amplitude relations are obtained. These nonlinear stable and unstable modes, which arise due to modal coupling and the symmetries of the models, control and explain the dynamics of the model under forced vibration. Based on these results, we study the behavior of the models subjected to a base excitation through a systematic study of the global and local bifurcations, and the integrity of stable solutions through the evolution and stratification of the basins of attraction and dynamic integrity measures. Finally, we study how to increase the safety of the structure through the control of global homoclinic and heteroclinic bifurcations. This thesis identifies a number of 
behaviors that are typical of the two models and can be understood as characteristic phenomena of structures exhibiting modal coupling. Thus the main contribution of this work is to identify certain characteristics and particular aspects of this class of structures, a first contribution to this research area.

\section{Keywords}

Modal coupling, imperfection sensitivity, dynamic integrity and nonlinear modes. 


\section{Sumário}

1 Introdução 35

1.1. Objetivo 40

1.2. Organização do Trabalho 41

2 Formulação do Problema 43

2.1. Modelo de Augusti 43

2.1.1. Energia Cinética 45

2.1.2. Energia Potencial Total 46

2.1.3. Amortecimento 47

2.1.4. Função de Lagrange 48

2.1.5. Equações de Movimento 49

2.2. Modelo de Torre Estaiada 49

2.2.1. Energia Cinética 50

2.2.2. Energia Potencial Total 51

2.2.3. Amortecimento 52

2.2.4. Função de Lagrange 52

2.2.5. Equações de Movimento 53

3 Análise Estática $\quad 54$

3.1. Modelo de Augusti 54

3.1.1. Modelo Perfeito 54

3.1.1.1. Caminhos Pós-Críticos 56

3.1.1.2. Superfícies de Energia 57

3.1.2. Influência da Rigidez Relativa das Molas 58

3.1.2.1. Caminhos Pós-Críticos $\quad 59$

3.1.2.2. Superfícies de Energia 60

3.1.3. Modelo com Imperfeição Geométrica 61

3.1.3.1. Caminhos Não-Lineares de Equilíbrio 62

3.1.3.2. Superfícies de Energia 65

3.2. Modelo de Torre Estaiada 66

3.2.1. Modelo Perfeito 66 
3.2.1.1. Caminhos Pós-Críticos 70

3.2.1.2. Superfícies de Energia $\quad 74$

3.2.2. Influência da Rigidez Relativa das Molas 76

3.2.2.1. Caminhos Pós-Críticos 78

3.2.2.2. Superfícies de Energia $\quad 79$

3.2.3. Modelo com Imperfeição Geométrica 80

3.2.3.1. Caminhos Não-Lineares de Equilíbrio 82

3.2.3.2. Superfícies de Energia 85

4 Análise Dinâmica - Vibração Livre 86

4.1. Modelo de Augusti 86

4.1.1. Freqüências Naturais $\quad 87$

4.1.2. Princípio da Conservação de Energia 94

4.1.3. Variedades Invariantes dos Pontos de Sela 99

4.1.4. Modos Não-Lineares de Vibração 103

$\begin{array}{ll}\text { 4.1.4.1. Modelo Perfeito } & 107\end{array}$

4.1.4.2. Influência da Rigidez Relativa das Molas 117

4.1.4.3. Modelo com Imperfeição Geométrica 121

4.2. Modelo de Torre Estaiada 130

4.2.1. Freqüências Naturais 132

4.2.2. Princípio da Conservação de Energia 140

4.2.3. Variedades Invariantes dos Pontos de Sela 144

4.2.4. Modos Não-Lineares de Vibração 148

4.2.4.1. Modelo Perfeito 149

4.2.4.2. Influência da Rigidez Relativa das Molas 155

4.2.4.3. Modelo com Imperfeição Geométrica 163

5 Análise Dinâmica - Vibração Forçada 172

5.1. Introdução 172

5.1.1. Fronteiras de Escape 172

5.1.2. Diagramas de Bifurcação 173

5.1.3. Bacias de Atração e Integridade Dinâmica 177

5.2. Modelo de Augusti 178

$\begin{array}{ll}\text { 5.2.1. Modelo Perfeito } & 180\end{array}$ 
5.2.2. Influência da Rigidez Relativa das Molas 200

5.2.3. Modelo com Imperfeição Geométrica 207

$\begin{array}{ll}\text { 5.3. Modelo de Torre Estaiada } & 214\end{array}$

5.3.1. Modelo Perfeito 217

5.3.2. Influência da Rigidez Relativa das Molas 229

5.3.3. Modelo com Imperfeição Geométrica 238

6 Controle da Erosão das Bacias de Atração 244

6.1. Introdução 244

6.1.1. Medidas de Integridade 244

6.1.2. Redução da Integridade 245

$\begin{array}{ll}\text { 6.1.3. Controle da Integridade } & 247\end{array}$

6.2. Equações de Movimento Desacopladas 250

6.2.1. Modelo de Augusti 250

6.2.2. Modelo de Torre Estaiada 251

6.3. Formulação do Controle 253

6.3.1. Bifurcações Globais 254

6.3.2. Controle a partir da Adição de Super-Harmônicos 260

6.3.3. Controle Ótimo 265

6.4. Aplicação do Controle Ótimo 267

6.4.1. Modelo de Augusti 267

6.4.1.1. Modelo Perfeito 267

6.4.1.2. Modelo com Imperfeição Geométrica 275

6.4.2. Modelo de Torre Estaiada 283

7 Conclusões e Sugestões $\quad 289$

7.1. Conclusões 289

$\begin{array}{ll}\text { 7.2. Sugestões } & 291\end{array}$

8 Referências Bibliográficas $\quad 292$ 


\section{Lista de Figuras}

Figura 2.1: Modelo de Augusti. 43

Figura 2.2: Modelo de Augusti imperfeito. 44

Figura 2.3: Modelo simplificado de torre estaiada. 49

Figura 3.1: Caminhos pós-críticos. Modelo de Augusti perfeito. 56

Figura 3.2: Projeções dos caminhos pós-críticos. Modelo de Augusti

perfeito. 56

Figura 3.3: Superfícies de energia potencial total. Modelo de Augusti

perfeito. 58

Figura 3.4: Caminhos pós-críticos. Modelo de Augusti considerando a influência da rigidez relativa das molas.

Figura 3.5: Projeções dos caminhos pós-críticos. Modelo de Augusti considerando a influência da rigidez relativa das molas.

Figura 3.6: Superfícies de energia potencial total para $\lambda=0.9$. Modelo de Augusti considerando a influência da rigidez relativa das molas.

Figura 3.7: Cortes nas superfícies de energia potencial total para $\lambda=0.9$. Modelo de Augusti considerando a influência da rigidez relativa das molas.

Figura 3.8: Caminhos não-lineares de equilíbrio para $\phi=1^{\circ} \mathrm{e} \psi=0^{\circ}$. Modelo de Augusti com imperfeição geométrica.

Figura 3.9: Projeções dos caminhos não-lineares de equilíbrio para $\phi=1^{\circ}$ e $\psi=0^{\circ}$. Modelo de Augusti com imperfeição geométrica.

Figura 3.10: Caminhos não-lineares de equilíbrio para $\phi=1^{\circ}$. Modelo de Augusti com imperfeição geométrica.

Figura 3.11: Projeções dos caminhos não-lineares de equilíbrio para $\phi=1^{\circ}$. Modelo de Augusti com imperfeição geométrica.

Figura 3.12: Variação da carga limite (ponto de bifurcação) com as grandezas que definem a imperfeição, $\phi$ e $\psi$. Modelo de Augusti com imperfeição geométrica.

Figura 3.13: Superfícies de energia potencial total para $\lambda=0.9$ e $\phi=1^{\circ}$. 
Modelo de Augusti com imperfeição geométrica.

Figura 3.14: Comportamento do modelo de torre estaiada em função do ângulo $\beta$ (Thompson \& Gaspar, 1977).

Figura 3.15: Caminhos pós-críticos para $\beta=75^{\circ}$ - caso monoclinal. Modelo de torre estaiada perfeito.

Figura 3.16: Projeções dos caminhos pós-críticos para $\beta=75^{\circ}$ - caso monoclinal. Modelo de torre estaiada perfeito.

Figura 3.17: Caminhos pós-críticos para $\beta=50^{\circ}$ - caso homeoclinal. Modelo de torre estaiada perfeito.

Figura 3.18: Projeções dos caminhos pós-críticos para $\beta=50^{\circ}$ - caso homeoclinal. Modelo de torre estaiada perfeito.

Figura 3.19: Caminhos pós-críticos para $\beta=120^{\circ}$ - caso anticlinal. Modelo de torre estaiada perfeito.

Figura 3.20: Projeções dos caminhos pós-críticos para $\beta=120^{\circ}$ - caso anticlinal. Modelo de torre estaiada perfeito.

Figura 3.21: Superfícies de energia potencial total para $\beta=75^{\circ}$ - caso monoclinal. Modelo de torre estaiada perfeito.

Figura 3.22: Superfícies de energia potencial total para $\beta=50^{\circ}$ - caso homeoclinal. Modelo de torre estaiada perfeito.

Figura 3.23: Superfícies de energia potencial total para $\beta=120^{\circ}$ - caso anticlinal. Modelo de torre estaiada perfeito.

Figura 3.24: Caminhos pós-críticos para $\beta=120^{\circ}$ - caso anticlinal.

Modelo de torre estaiada considerando a influência da rigidez relativa das molas.

Figura 3.25: Projeções dos caminhos pós-críticos para $\beta=120^{\circ}$ - caso anticlinal. Modelo de torre estaiada considerando a influência da rigidez relativa das molas.

Figura 3.26: Superfícies de energia potencial total para $\lambda=0.7$ e $\beta=120^{\circ}$ caso anticlinal. Modelo de torre estaiada considerando a influência da rigidez relativa das molas.

Figura 3.27: Caminhos não-lineares de equilíbrio para $\phi=1^{\circ}$ e $\beta=120^{\circ}$ caso anticlinal. Modelo de torre estaiada com imperfeição geométrica. 83 
Figura 3.28: Projeções dos caminhos não-lineares de equilíbrio para $\phi=1^{\circ}$ e $\beta=120^{\circ}$ - caso anticlinal. Modelo de torre estaiada com imperfeição geométrica.

Figura 3.29: Variação da carga limite (ponto de bifurcação) com as grandezas que definem a imperfeição, $\phi$ e $\psi$, para $\beta=120^{\circ}$ - caso anticlinal. Modelo de torre estaiada com imperfeição geométrica.

Figura 3.30: Superfícies de energia potencial total para $\phi=1^{\circ}, \lambda=0.7 \mathrm{e}$ $\beta=120^{\circ}$ - caso anticlinal. Modelo de torre estaiada com imperfeição geométrica.

Figura 4.1: Configurações do modelo de Augusti.

Figura 4.2: Variação da maior freqüência natural com o parâmetro de rigidez $\alpha$, para $\lambda=0.9$. Modelo de Augusti considerando a influência da rigidez relativa das molas.

Figura 4.3: Variação das freqüências naturais com os parâmetros $\psi \mathrm{e} \phi$, para $\lambda=0.9$. Modelo de Augusti com imperfeição geométrica.

Figura 4.4: Seções das bacias de atração conservativas em 3D $\left(\theta_{1} \times \theta_{2} \times \mathrm{d} \theta_{1} / \mathrm{dt}\right)$, para $\lambda=0.9$ e $\omega_{p}=1.0 / \mathrm{s}$. Modelo de Augusti.

Figura 4.5: Seções das bacias de atração conservativas em 3D ( $\mathrm{d} \theta_{1} / \mathrm{dt} x \mathrm{~d} \theta_{2} / \mathrm{dt} x \theta_{1}$ ), para $\lambda=0.9$ e $\omega_{p}=1.0 /$ s. Modelo de Augusti.

Figura 4.6: Seções das bacias de atração conservativas em 2D, para $\lambda=0.9$ e $\omega_{p}=1.0 / \mathrm{s}$. Modelo de Augusti.

Figura 4.7: Projeções das variedades invariantes dos pontos de sela, para $\lambda=0.9$ e $\omega_{p}=1.0 / \mathrm{s}$. Modelo de Augusti perfeito.

Figura 4.8: Projeções em planos de fase da reposta no tempo do primeiro ponto sela perturbado, para $\lambda=0.9$ e $\omega_{p}=1.0 / \mathrm{s}$. Modelo de Augusti perfeito.

Figura 4.9: Projeções das variedades invariantes e da reposta no tempo no plano $\theta_{1} \times \theta_{2}$, para $\alpha=1.15, \lambda=0.9$ e $\omega_{p}=1.0 / \mathrm{s}$. Modelo de Augusti considerando a influência da rigidez relativa das molas.

Figura 4.10: Projeções das variedades invariantes e da reposta no tempo no plano $\theta_{1} x \theta_{2}$, para $\psi=0^{\circ}, \phi=1^{\circ}, \lambda=0.9$ e $\omega_{p}=1.0 / \mathrm{s}$. Modelo de Augusti com imperfeição geométrica. 
Figura 4.11: Projeções das variedades invariantes e da reposta no tempo, para $\psi=45^{\circ}, \phi=1^{\circ}, \lambda=0.9$ e $\omega_{p}=1.0 / \mathrm{s}$. Modelo de Augusti com imperfeição geométrica.

Figura 4.12: Seções de Poincaré para $\omega_{1}=\omega_{2}=1 / 3, \lambda=0.9$ e $\omega_{p}=1.0 / \mathrm{s}$. Modelo de Augusti perfeito.

Figura 4.13: Comportamento no domínio do tempo dos pontos P01 e P02, para $\omega_{1}=\omega_{2}=1 / 3, \lambda=0.9$ e $\omega_{p}=1.0 /$ s. Modelo de Augusti perfeito.

Figura 4.14: Comportamento no domínio do tempo dos pontos

P11, P21, P12 e P22, para $\omega_{1}=\omega_{2}=1 / 3, \lambda=0.9$ e $\omega_{p}=1.0 / \mathrm{s}$.

Modelo de Augusti perfeito.

Figura 4.15: Seções de Poincaré dos pontos PS11, PQ11 e PC11, para $\omega_{1}=\omega_{2}=1 / 3, \lambda=0.9$ e $\omega_{p}=1.0 /$ s. Modelo de Augusti perfeito.

Figura 4.16: Coordenadas auxiliares, Modelo de Augusti perfeito.

Figura 4.17: Relações freqüência-amplitude para $\omega_{1}=\omega_{2}=1 / 3, \lambda=0.9 \mathrm{e}$ $\omega_{p}=1.0 /$ s. Modelo de Augusti perfeito.

Figura 4.18: Relações freqüência-amplitude dos modos acoplados instáveis dos pontos de sela PS11, PS21, PS12 e PS22, para $\omega_{1}=\omega_{2}=1 / 3, \lambda=0.9$ e $\omega_{p}=1.0 / \mathrm{s}$. Modelo de Augusti perfeito.

Figura 4.19: Seções de Poincaré com $5 \%$ da energia do ponto de sela, para $\alpha=1.3, \omega_{1}=1 / 3, \omega_{2}=2 / 3, \lambda=0.9$ e $\omega_{p}=1.0 / \mathrm{s}$. Modelo de Augusti considerando a influência da rigidez relativa das molas.

Figura 4.20: Comportamento no domínio do tempo dos pontos P01 e P02, para $\alpha=1.3, \omega_{1}=1 / 3, \omega_{2}=2 / 3, \lambda=0.9$ e $\omega_{p}=1.0 / \mathrm{s}$. Modelo de Augusti considerando a influência da rigidez relativa das molas.

Figura 4.21: Comportamento dos pontos P12, P22, P32 e P42, para $\alpha=1.3, \omega_{1}=1 / 3, \omega_{2}=2 / 3, \lambda=0.9$ e $\omega_{p}=1.0 / \mathrm{s}$. Modelo de Augusti considerando a influência da rigidez relativa das molas.

Figura 4.22: Relações freqüência-amplitude dos modos não-lineares estáveis desacoplados, para $\alpha=1.3, \omega_{1}=1 / 3, \omega_{2}=2 / 3, \lambda=0.9$ e $\omega_{p}=1.0 / \mathrm{s}$. Modelo de Augusti considerando a influência da rigidez relativa das molas. 
Figura 4.23: Seções de Poincaré para $\psi=0^{\circ}, \phi=1^{\circ}, \omega_{1}=0.311, \omega_{2}=0.353$, $\lambda=0.9$ e $\omega_{p}=1.0 / \mathrm{s}$. Modelo de Augusti com imperfeição geométrica.

Figura 4.24: Comportamento no domínio do tempo dos pontos P01 e P02, para $\psi=0^{\circ}, \phi=1^{\circ}, \omega_{1}=0.311, \omega_{2}=0.353, \lambda=0.9$ e $\omega_{p}=1.0 / \mathrm{s}$. Modelo de Augusti com imperfeição geométrica.

Figura 4.25: Relação freqüência-amplitude do modo não-linear estável desacoplado no plano $\theta_{D 1} x \mathrm{~d} \theta_{D 1} / \mathrm{dt}$, para $\psi=0^{\circ}, \phi=1^{\circ}, \omega_{1}=0.311$, $\omega_{2}=0.353, \lambda=0.9$ e $\omega_{p}=1.0 / \mathrm{s}$. Modelo de Augusti com imperfeição geométrica.

Figura 4.26: Relação freqüência-amplitude do modo acoplado estável do ponto P01, para $\psi=0^{\circ}, \phi=1^{\circ}, \omega_{1}=0.311, \omega_{2}=0.353, \lambda=0.9$ e $\omega_{p}=1.0 / \mathrm{s}$. Modelo de Augusti com imperfeição geométrica.

Figura 4.27: Seções de Poincaré com 50 \% da energia do ponto de sela, para $\psi=45^{\circ}, \phi=1^{\circ}, \omega_{1}=0.302, \omega_{2}=0.361, \lambda=0.9$ e $\omega_{p}=1.0 / \mathrm{s}$. Modelo de Augusti com imperfeição geométrica.

Figura 4.28: Seção de Poincaré com $50 \%$ da energia do ponto de sela no plano $\theta_{D 1} \times \theta_{D 2}$, para $\psi=45^{\circ}, \phi=1^{\circ}, \omega_{1}=0.302, \omega_{2}=0.361, \lambda=0.9 \mathrm{e}$ $\omega_{p}=1.0 /$ s. Modelo de Augusti com imperfeição geométrica.

Figura 4.29: Comportamento no domínio do tempo dos pontos P11, P21, P12 e P22, para $\psi=45^{\circ}, \phi=1^{\circ}, \omega_{1}=0.302, \omega_{2}=0.361, \lambda=0.9$ e $\omega_{p}=1.0 / \mathrm{s}$. Modelo de Augusti com imperfeição geométrica.

Figura 4.30: Coordenadas auxiliares considerando $\psi=45^{\circ}$. Modelo de Augusti com imperfeição geométrica.

Figura 4.31: Relações freqüência-amplitude dos modos não-lineares nãosimilares estáveis acoplados dos pontos P21 e P12, para $\psi=45^{\circ}, \phi=1^{\circ}$, $\omega_{1}=0.302, \omega_{2}=0.361, \lambda=0.9$ e $\omega_{p}=1.0 / \mathrm{s}$. Modelo de Augusti com imperfeição geométrica.

Figura 4.32: Relação freqüência-amplitude dos modos não-lineares similares estáveis acoplados dos pontos $\mathrm{P} 11$ e $\mathrm{P} 12$, para $\psi=45^{\circ}, \phi=1^{\circ}$, $\omega_{1}=0.302, \omega_{2}=0.361, \lambda=0.9$ e $\omega_{p}=1.0 / \mathrm{s}$. Modelo de Augusti com imperfeição geométrica.

Figura 4.33: Variação das freqüências naturais em função de rigidez $\alpha$, 
para $\lambda=0.7$ e $\beta=120^{\circ}$. Modelo de torre estaiada considerando a influência da rigidez relativa das molas.

Figura 4.34: Variação das freqüências naturais com os parâmetros $\psi \mathrm{e} \phi$, para $\lambda=0.7$ e $\beta=120^{\circ}$. Modelo de torre estaiada com imperfeição geométrica.

Figura 4.35: Seções das bacias de atração conservativas em 3D $\left(u_{1} \times u_{2} x \mathrm{~d} u_{1} / \mathrm{dt}\right)$, para $\omega_{p}=1.0 / \mathrm{s}, \lambda=0.7$ e $\beta=120^{\circ}$. Modelo de torre estaiada.

Figura 4.36: Seções das bacias de atração conservativas em 2D, para $\omega_{p}=1.0 / \mathrm{s}, \lambda=0.7$ e $\beta=120^{\circ}$. Modelo de torre estaiada.

Figura 4.37: Projeções das variedades invariantes dos pontos de sela, para $\lambda=0.7, \beta=120^{\circ}$ e $\omega_{p}=1.0 /$ s. Modelo de torre estaiada perfeito. 145 Figura 4.38: Projeções da reposta no tempo do primeiro ponto sela perturbado, para $\lambda=0.7, \beta=120^{\circ}$ e $\omega_{p}=1.0 / \mathrm{s}$. Modelo de torre estaiada perfeito.

Figura 4.39: Projeções das variedades invariantes e da reposta no tempo no plano $u_{1} x u_{2}$, para $\alpha=0.82, \lambda=0.7, \beta=120^{\circ}$ e $\omega_{p}=1.0 / \mathrm{s}$. Modelo de torre estaiada considerando a influência da rigidez relativa das molas. 146 Figura 4.40: Projeções das variedades invariantes e da reposta no tempo, para $\alpha=1.18, \lambda=0.7, \beta=120^{\circ}$ e $\omega_{p}=1.0 / \mathrm{s}$. Modelo de torre estaiada considerando a influência da rigidez relativa das molas.

Figura 4.41: Projeções das variedades invariantes e da reposta no tempo no plano $u_{1} x u_{2}$, para $\psi=0^{\circ}, \phi=1^{\circ}, \lambda=0.7, \beta=120^{\circ}$ e $\omega_{p}=1.0 /$ s. Modelo de torre estaiada com imperfeição geométrica.

Figura 4.42: Projeções das variedades invariantes e da reposta no tempo no plano $u_{1} x u_{2}$, para $\psi=90^{\circ}, \phi=1^{\circ}, \lambda=0.7, \beta=120^{\circ}$ e $\omega_{p}=1.0 /$ s. Modelo de torre estaiada com imperfeição geométrica.

Figura 4.43: Seções de Poincaré com 50 \% da energia do ponto de sela, para $\omega_{1}=\omega_{2}=0.655, \lambda=0.7, \beta=120^{\circ}$ e $\omega_{p}=1.0 / \mathrm{s}$. Modelo de torre estaiada perfeito.

Figura 4.44: Comportamento no domínio do tempo dos pontos P01, P11, $\mathrm{P} 21, \mathrm{P} 31, \mathrm{P} 41, \mathrm{P} 12, \mathrm{P} 22, \mathrm{P} 32$ e P42, para $\omega_{1}=\omega_{2}=0.655, \lambda=0.7, \beta=120^{\circ}$ 
e $\omega_{p}=1.0 / \mathrm{s}$. Modelo de torre estaiada perfeito.

Figura 4.45: Coordenadas auxiliares. Modelo de torre estaiada perfeito.

Figura 4.46: Relação freqüência-amplitude do modo não-linear estável desacoplado no plano $u_{2} x d u_{2} / d t$, para $\omega_{1}=\omega_{2}=0.655, \lambda=0.7, \beta=120^{\circ}$ e $\omega_{p}=1.0 / \mathrm{s}$. Modelo de torre estaiada perfeito.

Figura 4.47: Relações freqüência-amplitude dos modos acoplados instáveis dos pontos de sela PS11, PS21 e PS12, para $\omega_{1}=\omega_{2}=0.655$, $\lambda=0.7, \beta=120^{\circ}$ e $\omega_{p}=1.0 / \mathrm{s}$. Modelo de torre estaiada perfeito.

Figura 4.48: Relações freqüência-amplitude dos modos não-lineares nãosimilares estáveis acoplados associados aos pontos P31, P41, P32 e $\mathrm{P} 42$, para $\omega_{1}=\omega_{2}=0.655, \lambda=0.7, \beta=120^{\circ}$ e $\omega_{p}=1.0 / \mathrm{s}$. Modelo de torre estaiada perfeito.

Figura 4.49: Relação freqüência-amplitude dos modos não-lineares similares estáveis acoplados dos pontos P11, P21, P12 e P22, para $\omega_{1}=\omega_{2}=0.655, \lambda=0.7, \beta=120^{\circ}$ e $\omega_{p}=1.0 / \mathrm{s}$. Modelo de torre estaiada perfeito.

Figura 4.50: Seções de Poincaré com 50 \% da energia do ponto de sela, para $\alpha=0.82, \omega_{1}=0.414, \omega_{2}=0.828, \lambda=0.7, \beta=120^{\circ}$ e $\omega_{p}=1.0 / \mathrm{s}$. Modelo de torre estaiada considerando a influência da rigidez relativa das molas.

Figura 4.51: Comportamento no domínio do tempo dos pontos P11, P21, $\mathrm{P} 31, \mathrm{P} 41, \mathrm{P} 12$ e P22, para $\alpha=0.82, \omega_{1}=0.414, \omega_{2}=0.828, \lambda=0.7$, $\beta=120^{\circ}$ e $\omega_{p}=1.0 / \mathrm{s}$. Modelo de torre estaiada considerando a influência da rigidez relativa das molas.

Figura 4.52: Relações freqüência-amplitude do modo desacoplado instável do ponto de sela PS01, para $\alpha=0.82, \omega_{1}=0.414, \omega_{2}=0.828$, $\lambda=0.7, \beta=120^{\circ}$ e $\omega_{p}=1.0 / \mathrm{s}$. Modelo de torre estaiada considerando a influência da rigidez relativa das molas.

Figura 4.53: Relações freqüência-amplitude dos modos não-lineares acoplados estáveis, para $\alpha=0.82, \omega_{1}=0.414, \omega_{2}=0.828, \lambda=0.7, \beta=120^{\circ} \mathrm{e}$ $\omega_{p}=1.0 / \mathrm{s}$. Modelo de torre estaiada considerando a influência da rigidez 
relativa das molas.

Figura 4.54: Seções de Poincaré com 50 \% da energia do ponto de sela, para $\alpha=1.18, \omega_{1}=0.414, \omega_{2}=0.828, \lambda=0.7, \beta=120^{\circ}$ e $\omega_{p}=1.0 / \mathrm{s}$. Modelo de torre estaiada considerando a influência da rigidez relativa das molas.

Figura 4.55: Comportamento no domínio do tempo dos pontos P01, P11, P21, P12, P22, P32, P42 e P52, para $\alpha=1.18, \omega_{1}=0.414, \omega_{2}=0.828$, $\lambda=0.7, \beta=120^{\circ}$ e $\omega_{p}=1.0 / \mathrm{s}$. Modelo de torre estaiada considerando a influência da rigidez relativa das molas.

Figura 4.56: Relação freqüência-amplitude do modo não-linear estável desacoplado no plano $u_{2} x d u_{2} / d t$, P01, para $\alpha=1.18, \omega_{1}=0.414, \omega_{2}=0.828$, $\lambda=0.7, \beta=120^{\circ}$ e $\omega_{p}=1.0 / \mathrm{s}$. Modelo de torre estaiada considerando a influência da rigidez relativa das molas.

Figura 4.57: Relações freqüência-amplitude dos modos não-lineares acoplados estáveis, para $\alpha=1.18, \omega_{1}=0.414, \omega_{2}=0.828, \lambda=0.7, \beta=120^{\circ} \mathrm{e}$ $\omega_{p}=1.0 / \mathrm{s}$. Modelo de torre estaiada considerando a influência da rigidez relativa das molas.

Figura 4.58: Seções de Poincaré com $50 \%$ da energia do ponto de sela, para $\psi=0^{\circ}, \phi=1^{\circ}, \omega_{1}=0.609, \omega_{2}=0.697, \lambda=0.7, \beta=120^{\circ}$ e $\omega_{p}=1.0 / \mathrm{s}$. Modelo de torre estaiada com imperfeição geométrica.

Figura 4.59: Comportamento no domínio do tempo dos pontos P11, P21, $\mathrm{P} 12$ e P22, para $\psi=0^{\circ}, \phi=1^{\circ}, \omega_{1}=0.609, \omega_{2}=0.697, \lambda=0.7, \beta=120^{\circ} \mathrm{e}$ $\omega_{p}=1.0 / \mathrm{s}$. Modelo de torre estaiada com imperfeição geométrica.

Figura 4.60: Relações freqüência-amplitude dos modos acoplados associados aos pontos P11, P21, P12 e P22, para $\psi=0^{\circ}, \phi=1^{\circ}$, $\omega_{1}=0.609, \omega_{2}=0.697, \lambda=0.7, \beta=120^{\circ}$ e $\omega_{p}=1.0 / \mathrm{s}$. Modelo de torre estaiada com imperfeição geométrica.

Figura 4.61: Seções de Poincaré para $\psi=90^{\circ}, \phi=1^{\circ}, \omega_{1}=0.612$, $\omega_{2}=0.693, \lambda=0.7, \beta=120^{\circ}$ e $\omega_{p}=1.0 / \mathrm{s}$. Modelo de torre estaiada com imperfeição geométrica.

Figura 4.62: Comportamento no domínio do tempo dos pontos P01 e P12 (P12'), para $\psi=90^{\circ}, \phi=1^{\circ}, \omega_{1}=0.612, \omega_{2}=0.693, \lambda=0.7, \beta=120^{\circ} \mathrm{e}$ 
$\omega_{p}=1.0 / \mathrm{s}$. Modelo de torre estaiada com imperfeição geométrica.

Figura 4.63: Relação freqüência-amplitude do modo não-linear estável desacoplado no plano $u_{2} \times d u_{2} / d t$, para $\psi=90^{\circ}, \phi=1^{\circ}, \omega_{1}=0.612$, $\omega_{2}=0.693, \lambda=0.7, \beta=120^{\circ}$ e $\omega_{p}=1.0 / \mathrm{s}$. Modelo de torre estaiada com imperfeição geométrica.

Figura 4.64: Relação freqüência-amplitude do modo não-linear acoplado estável associado ao ponto P12 (P12'), para $\psi=90^{\circ}, \phi=1^{\circ}, \omega_{1}=0.612$, $\omega_{2}=0.693, \lambda=0.7, \beta=120^{\circ}$ e $\omega_{p}=1.0 / \mathrm{s}$. Modelo de torre estaiada com imperfeição geométrica.

Figura 5.1: Forma como os multiplicadores de Floquet podem ultrapassar o círculo de raio unitário (região de estabilidade).

Figura 5.2: Bifurcação do tipo pitchfork, supercrítica e subcrítica.

Figura 5.3: Bifurcação do tipo nó-sela.

Figura 5.4: Bifurcação por duplicação de período, supercrítica e subcrítica.

Figura 5.5: Bifurcação do tipo Hopf, supercrítica e subcrítica.

Figura 5.6: Exemplo de diagrama de bifurcação.

Figura 5.7: Vista superior ilustrativa do Modelo de Augusti.

Figura 5.8: Fronteiras de estabilidade (escape) para $\lambda=0.9$ e $\xi_{1}=\xi_{2}=0.01$. Modelo de Augusti perfeito.

Figura 5.9: Variação da carga de escape, $F_{\text {esc }}$, com a direção da excitação, $\varphi$, (gráfico em coordenadas polares) para $\lambda=0.9 \mathrm{e}$ $\xi_{1}=\xi_{2}=0.01$. Modelo de Augusti perfeito.

Figura 5.10: Curvas de ressonância para $F=0.02, \lambda=0.9$ e $\xi_{1}=\xi_{2}=0.01$. Modelo de Augusti perfeito.

Figura 5.11: Curvas de ressonância e as relações freqüência-amplitude dos modos não-lineares, para $F=0.02, \lambda=0.9$ e $\xi_{1}=\xi_{2}=0.01$. Modelo de Augusti perfeito.

Figura 5.12: Fronteiras de estabilidade (escape), modelo acoplado e desacoplado, para $\varphi=0^{\circ}, \lambda=0.9$ e $\xi_{1}=\xi_{2}=0.01$. Modelo de Augusti perfeito (valores mínimos $-F_{\text {esc }}=0.0267$ (acoplado) e $F_{\text {esc }}=0.5800$ (desacoplado)). 
Figura 5.13: Curvas de ressonância, modelo acoplado e desacoplado, para $F=0.02, \varphi=0^{\circ}, \lambda=0.9$ e $\xi_{1}=\xi_{2}=0.01$. Modelo de Augusti perfeito.

Figura 5.14: Curvas de ressonância, modelo acoplado e desacoplado, para $F=0.03, \varphi=0^{\circ}, \lambda=0.9$ e $\xi_{1}=\xi_{2}=0.01$. Modelo de Augusti perfeito.

Figura 5.15: Diagramas de bifurcação, modelo acoplado e desacoplado, para $\Omega=1 / 3, \varphi=0^{\circ}, \lambda=0.9$ e $\xi_{1}=\xi_{2}=0.01$. Modelo de Augusti perfeito.

Figura 5.16: Respostas no tempo para dois níveis de carregamento, considerando diferentes condições inicias $\left(\theta_{1}, \mathrm{~d} \theta_{1} / \mathrm{dt}, \theta_{2}, \mathrm{~d} \theta_{2} / \mathrm{dt}\right)$, para $\Omega=1 / 3$ e $\varphi=0^{\circ}, \lambda=0.9$ e $\xi_{1}=\xi_{2}=0.01$. Modelo de Augusti perfeito.

Figura 5.17: Diagramas de bifurcação, modelo acoplado e desacoplado, para $\varphi=0^{\circ}, \lambda=0.9$ e $\xi_{1}=\xi_{2}=0.01$. Modelo de Augusti perfeito.

Figura 5.18: Seções das bacias de atração no plano $\theta_{1} \times d \theta_{1} / d t$, modelo acoplado e desacoplado, para $\Omega=0.525, \varphi=0^{\circ}, \lambda=0.9$ e $\xi_{1}=\xi_{2}=0.01$. Modelo de Augusti perfeito.

Figura 5.19: Seções das bacias de atração no plano $\theta_{1} \times d \theta_{1} / d t$, modelo acoplado e desacoplado, para $\Omega=1 / 3, F=0.1, \varphi=0^{\circ}, \lambda=0.9 \mathrm{e}$ $\xi_{1}=\xi_{2}=0.01$. Modelo de Augusti perfeito.

Figura 5.20: Seções das bacias de atração no plano $\theta_{1} \times \theta_{2}$, modelo acoplado, para $\Omega=1 / 3, \varphi=0^{\circ}, \lambda=0.9$ e $\xi_{1}=\xi_{2}=0.01$. Modelo de Augusti perfeito.

Figura 5.21: Medida de integridade local da bacia de atração, LIM, modelo acoplado e desacoplado, para $\Omega=1 / 3, \varphi=0^{\circ}, \lambda=0.9$ e $\xi_{1}=\xi_{2}=0.01$. Modelo de Augusti perfeito.

Figura 5.22: Fronteiras de estabilidade (escape), modelo acoplado e desacoplado, para $\varphi=45^{\circ}, \lambda=0.9$ e $\xi_{1}=\xi_{2}=0.01$. Modelo de Augusti perfeito (valores mínimos $-F_{\text {esc }}=0.07$ (acoplado e desacoplado)).

Figura 5.23: Mapeamento das bifurcações locais na região de ressonância fundamental, modelo acoplado e desacoplado, para $\varphi=45^{\circ}$, $\lambda=0.9$ e $\xi_{1}=\xi_{2}=0.01$. Modelo de Augusti perfeito. 
Figura 5.24: Diagramas de bifurcação, modelo acoplado e desacoplado, para $\Omega=0.4, \varphi=45^{\circ}, \lambda=0.9$ e $\xi_{1}=\xi_{2}=0.01$. Modelo de Augusti perfeito.

Figura 5.25: Diagramas de bifurcação, modelo acoplado e desacoplado, para $\Omega=1 / 3, \varphi=45^{\circ}, \lambda=0.9$ e $\xi_{1}=\xi_{2}=0.01$. Modelo de Augusti perfeito.

Figura 5.26: Seções das bacias de atração nos planos $\theta_{1} \times d \theta_{1} / d t$ e $\theta_{1} \times \theta_{2}$, modelo acoplado, para $\Omega=1 / 3, \varphi=45^{\circ}, \lambda=0.9$ e $\xi_{1}=\xi_{2}=0.01$. Modelo de Augusti perfeito.

Figura 5.27: Seções das bacias de atração e variedades dos pontos de sela no plano $u x d u / d t$, modelo desacoplado, para $\Omega=1 / 3, \varphi=45^{\circ}, \lambda=0.9 \mathrm{e}$ $\xi_{1}=\xi_{2}=0.01$. Modelo de Augusti perfeito.

Figura 5.28: Medida de integridade local da bacia de atração, LIM, modelo acoplado e desacoplado, para $\varphi=45^{\circ}, \lambda=0.9$ e $\xi_{1}=\xi_{2}=0.01$. Modelo de Augusti perfeito.

Figura 5.29: Mapeamento das bifurcações locais na região de ressonância fundamental, modelo acoplado, para $\varphi=2^{\circ}, \lambda=0.9 \mathrm{e}$ $\xi_{1}=\xi_{2}=0.01$. Modelo de Augusti perfeito (valores mínimos (2 vales) $-F_{\text {esc }}$ $=0.2705$ e $F_{\text {esc }}=0.0882$ ).

Figura 5.30: Diagramas de bifurcação, modelo acoplado, para $\Omega=0.3$, $\varphi=2^{\circ}, \lambda=0.9$ e $\xi_{1}=\xi_{2}=0.01$. Modelo de Augusti perfeito $\left(F_{\text {esc }}=0.3545\right)$.

Figura 5.31: Variação da medida de integridade local da bacia de atração, LIM, modelo acoplado, para $\Omega=1 / 3, \lambda=0.9$ e $\xi_{1}=\xi_{2}=0.01$. Modelo de Augusti perfeito.

Figura 5.32: Fronteiras de estabilidade (escape) para $\lambda=0.9 \mathrm{e}$ $\xi_{1}=\xi_{2}=0.01$. Modelo de Augusti considerando a influência da rigidez relativa das molas.

Figura 5.33: Variação da carga de escape, $F_{e s c}$, com a direção da excitação, $\varphi$, (gráfico em coordenadas polares) para $\lambda=0.9 \mathrm{e}$ $\xi_{1}=\xi_{2}=0.01$. Modelo de Augusti considerando a influência da rigidez relativa das molas. 
Figura 5.34: Curvas de ressonância para $F=0.02, \alpha=1.3, \lambda=0.9 \mathrm{e}$ $\xi_{1}=\xi_{2}=0.01$. Modelo de Augusti considerando a influência da rigidez relativa das molas.

Figura 5.35: Fronteiras de estabilidade (escape), modelo acoplado e desacoplado, para $\alpha=1.3, \lambda=0.9$ e $\xi_{1}=\xi_{2}=0.01$. Modelo de Augusti considerando a influência da rigidez relativa das molas.

Figura 5.36: Mapeamento das bifurcações locais na região de ressonância fundamental, modelo acoplado, para $\alpha=1.3, \lambda=0.9 \mathrm{e}$ $\xi_{1}=\xi_{2}=0.01$. Modelo de Augusti considerando a influência da rigidez relativa das molas.

Figura 5.37: Diagramas de bifurcação, modelo acoplado, para $\Omega=2 / 3$, $\varphi=45^{\circ}, \alpha=1.3, \lambda=0.9$ e $\xi_{1}=\xi_{2}=0.01$. Modelo de Augusti considerando a influência da rigidez relativa das molas $\left(F_{\text {esc }}=0.1315\right)$. 205 Figura 5.38: Seções das bacias de atração nos planos $\theta_{1} \times d \theta_{1} / d t, \theta_{2} \times d \theta_{2} / d t$ e $\theta_{1} \times \theta_{2}$, modelo acoplado, para $\Omega=2 / 3, \varphi=45^{\circ}, \alpha=1.3, \lambda=0.9 \mathrm{e}$ $\xi_{1}=\xi_{2}=0.01$. Modelo de Augusti considerando a influência da rigidez relativa das molas.

Figura 5.39: Variação da medida de integridade local da bacia de atração, LIM, modelo acoplado, para $\Omega=2 / 3, \alpha=1.3, \lambda=0.9$ e $\xi_{1}=\xi_{2}=0.01$. Modelo de Augusti considerando a influência da rigidez relativa das molas.

Figura 5.40: Fronteiras de estabilidade (escape) para $\lambda=0.9 \mathrm{e}$ $\xi_{1}=\xi_{2}=0.01$. Modelo de Augusti com imperfeição geométrica. 208 Figura 5.41: Variação da carga de escape, $F_{e s c}$, com a direção da excitação, $\varphi$, (gráfico em coordenadas polares) para $\lambda=0.9 \mathrm{e}$ $\xi_{1}=\xi_{2}=0.01$. Modelo de Augusti com imperfeição geométrica. Figura 5.42: Curvas de ressonância para $F=0.01, \phi=1^{\circ}, \psi=0^{\circ}, \lambda=0.9 \mathrm{e}$ $\xi_{1}=\xi_{2}=0.01$. Modelo de Augusti com imperfeição geométrica. 209

Figura 5.43: Curvas de ressonância para $F=0.01, \phi=1^{\circ}, \lambda=0.9 \mathrm{e}$ $\xi_{1}=\xi_{2}=0.01$. Modelo de Augusti com imperfeição geométrica. Figura 5.44 Mapeamento das bifurcações locais na região de ressonância fundamental, modelo acoplado e desacoplado, para $\varphi=45^{\circ}, \phi=1^{\circ}$, 
$\psi=45^{\circ}, \lambda=0.9$ e $\xi_{1}=\xi_{2}=0.01$. Modelo de Augusti com imperfeição geométrica.

Figura 5.45: Comparação do comportamento na região de ressonância fundamental entre o modelo perfeito e com imperfeição geométrica ( $\phi=1^{\circ}$ e $\psi=45^{\circ}$ ), modelo acoplado, para $\varphi=45^{\circ}, \lambda=0.9$ e $\xi_{1}=\xi_{2}=0.01$. (Valores mínimos $-F_{\text {esc }}=0.1653$ (perfeito) e $F_{\text {esc }}=0.0510$ (imperfeito)).

Figura 5.46: Seções das bacias de atração no plano $u x d u / d t$, modelo desacoplado, para $\Omega=0.3026, \varphi=45^{\circ}, \lambda=0.9$ e $\xi_{1}=\xi_{2}=0.01$. Modelo de Augusti.

Figura 5.47: Medida de integridade local da bacia de atração, LIM, modelo acoplado e desacoplado, para $\Omega=0.3026, \varphi=45^{\circ}, \phi=1^{\circ}, \psi=45^{\circ}, \lambda=0.9 \mathrm{e}$ $\xi_{1}=\xi_{2}=0.01$. Modelo de Augusti com imperfeição geométrica.

Figura 5.48: Comparação da medida de integridade local da bacia de atração, LIM, na ressonância fundamental entre o modelo perfeito e com imperfeição geométrica $\left(\phi=1^{\circ}\right.$ e $\left.\psi=45^{\circ}\right)$, modelo acoplado, para $\varphi=45^{\circ}$, $\lambda=0.9$ e $\xi_{1}=\xi_{2}=0.01$. 214

Figura 5.49 Vista superior ilustrativa do modelo de torre estaiada.

Figura 5.50: Fronteiras de estabilidade (escape) para $\lambda=0.7, \beta=120^{\circ} \mathrm{e}$ $\xi_{1}=\xi_{2}=0.01$. Modelo de torre estaiada perfeito.

Figura 5.51: Variação da carga de escape, $F_{e s c}$, com a direção da excitação, $\varphi$, (gráfico em coordenadas polares) para $\lambda=0.7, \beta=120^{\circ} \mathrm{e}$ $\xi_{1}=\xi_{2}=0.01$. Modelo de torre estaiada perfeito.

Figura 5.52: Curvas de ressonância para $F=0.02, \lambda=0.7, \beta=120^{\circ} \mathrm{e}$ $\xi_{1}=\xi_{2}=0.01$. Modelo de torre estaiada perfeito.

Figura 5.53: Curvas de ressonância para valores crescentes de $F, \varphi=0^{\circ}$, $\lambda=0.7, \beta=120^{\circ}$ e $\xi_{1}=\xi_{2}=0.01$. Modelo de torre estaiada perfeito.

Figura 5.54: Fronteiras de estabilidade (escape), modelo acoplado e desacoplado, para $\varphi=90^{\circ}, \lambda=0.7, \beta=120^{\circ}$ e $\xi_{1}=\xi_{2}=0.01$. Modelo de torre estaiada perfeito (valores mínimos $-F_{\text {esc }}=0.0200$ (acoplado) e $F_{\text {esc }}$ $=0.0400$ (desacoplado)).

Figura 5.55: Curvas de ressonância, modelo acoplado e desacoplado, para $F=0.002, \varphi=90^{\circ}, \lambda=0.7, \beta=120^{\circ}$ e $\xi_{1}=\xi_{2}=0.01$. Modelo de torre 
estaiada perfeito.

Figura 5.56: Curvas de ressonância, modelo acoplado e desacoplado, para $F=0.01, \varphi=90^{\circ}, \lambda=0.7, \beta=120^{\circ}$ e $\xi_{1}=\xi_{2}=0.01$. Modelo de torre estaiada perfeito.

Figura 5.57: Mapeamento das bifurcações locais na região de ressonância fundamental, modelo acoplado e desacoplado, para $\varphi=90^{\circ}$, $\lambda=0.7, \beta=120^{\circ}$ e $\xi_{1}=\xi_{2}=0.01$. Modelo de torre estaiada perfeito.

Figura 5.58: Diagramas de bifurcação, modelo acoplado e desacoplado, para $\Omega=0.6546, \varphi=90^{\circ}, \lambda=0.7, \beta=120^{\circ}$ e $\xi_{1}=\xi_{2}=0.01$. Modelo de torre estaiada perfeito.

Figura 5.59: Fronteiras de estabilidade (escape), modelo acoplado e desacoplado, para $\varphi=30^{\circ}, \lambda=0.7, \beta=120^{\circ}$ e $\xi_{1}=\xi_{2}=0.01$. Modelo de torre estaiada perfeito (valores mínimos $-F_{\text {esc }}=0.0250$ (acoplado) e $F_{\text {esc }}$ $=0.0300$ (desacoplado)).

Figura 5.60: Curvas de ressonância, modelo acoplado e desacoplado, para $F=0.02, \varphi=30^{\circ}, \lambda=0.7, \beta=120^{\circ}$ e $\xi_{1}=\xi_{2}=0.01$. Modelo de torre estaiada perfeito.

Figura 5.61: Mapeamento das bifurcações locais na região de ressonância fundamental, modelo acoplado e desacoplado, para $\varphi=30^{\circ}$, $\lambda=0.7, \beta=120^{\circ}$ e $\xi_{1}=\xi_{2}=0.01$. Modelo de torre estaiada perfeito.

Figura 5.62: Diagramas de bifurcação, modelo acoplado e desacoplado, para $\Omega=0.6546, \varphi=30^{\circ}, \lambda=0.7, \beta=120^{\circ}$ e $\xi_{1}=\xi_{2}=0.01$. Modelo de torre estaiada perfeito.

Figura 5.63: Seções das bacias de atração nos planos $u_{1} x d u_{1} / d t, u_{2} x d u_{2} / d t$ e $u_{1} x u_{2}$, modelo acoplado, para $\Omega=0.6546, \varphi=30^{\circ}, \lambda=0.7, \beta=120^{\circ} \mathrm{e}$ $\xi_{1}=\xi_{2}=0.01$. Modelo de torre estaiada perfeito.

Figura 5.64: Mapeamento das bifurcações locais na região de ressonância fundamental, modelo acoplado, para $\varphi=0^{\circ}, \lambda=0.7, \beta=120^{\circ} \mathrm{e}$ $\xi_{1}=\xi_{2}=0.01$. Modelo de torre estaiada perfeito (valores mínimos (2 vales) $-F_{\text {esc }}=0.0495$ e $F_{\text {esc }}=0.0150$ ).

Figura 5.65 Fronteiras de estabilidade (escape) para $\alpha<1, \lambda=0.7$, $\beta=120^{\circ}$ e $\xi_{1}=\xi_{2}=0.01$. Modelo de torre estaiada considerando a influência 
da rigidez relativa das molas.

Figura 5.66: Curvas de ressonância para $F=0.01, \alpha=0.82, \lambda=0.7$, $\beta=120^{\circ}$ e $\xi_{1}=\xi_{2}=0.01$. Modelo de torre estaiada considerando a influência da rigidez relativa das molas.

Figura 5.67: Mapeamento das bifurcações locais na região de ressonância fundamental, modelo acoplado e desacoplado, para $\alpha=0.82$, $\lambda=0.7, \beta=120^{\circ}$ e $\xi_{1}=\xi_{2}=0.01$. Modelo considerando a influência da rigidez relativa das molas.

Figura 5.68: Diagrama de bifurcação para $\Omega=0.828, \varphi=45^{\circ}, \alpha=0.82$, $\lambda=0.7, \beta=120^{\circ}$ e $\xi_{1}=\xi_{2}=0.01$. Modelo de torre estaiada considerando a influência da rigidez relativa das molas $\left(F_{\text {esc }}=0.0437\right)$.

Figura 5.69: Seções das bacias de atração nos planos $u_{1} x d u_{1} / d t, u_{2} x d u_{2} / d t$ e $u_{1} x u_{2}$, modelo acoplado, para $\Omega=0.828, \varphi=45^{\circ}, \alpha=0.82, \lambda=0.7$, $\beta=120^{\circ}$ e $\xi_{1}=\xi_{2}=0.01$. Modelo de torre estaiada considerando a influência da rigidez relativa das molas.

Figura 5.70: Medida de integridade local da bacia de atração, LIM, modelo acoplado, para $\Omega=0.828, \varphi=45^{\circ}, \alpha=0.82, \lambda=0.7, \beta=120^{\circ}$ e $\xi_{1}=\xi_{2}=0.01$. Modelo de torre estaiada considerando a influência da rigidez relativa das molas.

Figura 5.71: Fronteiras de estabilidade (escape) para $\alpha>1, \lambda=0.7$, $\beta=120^{\circ}$ e $\xi_{1}=\xi_{2}=0.01$. Modelo de torre estaiada considerando a influência da rigidez relativa das molas.

Figura 5.72: Variação da carga de escape, $F_{\text {esc }}$, com a direção da excitação, $\varphi$, (gráfico em coordenadas polares) para $\lambda=0.7, \beta=120^{\circ} \mathrm{e}$ $\xi_{1}=\xi_{2}=0.01$. Modelo de torre estaiada considerando a influência da rigidez relativa das molas.

Figura 5.73: Curvas de ressonância para $F=0.01, \alpha=1.18, \lambda=0.7$, $\beta=120^{\circ}$ e $\xi_{1}=\xi_{2}=0.01$. Modelo de torre estaiada considerando a influência da rigidez relativa das molas.

Figura 5.74: Fronteiras de estabilidade (escape), modelo acoplado e desacoplado, para $\varphi=90^{\circ}, \alpha=1.18, \lambda=0.7, \beta=120^{\circ}$ e $\xi_{1}=\xi_{2}=0.01$. Modelo de torre estaiada considerando a influência da rigidez relativa das 
molas (valores mínimos $-F_{\text {esc }}=0.047$ (acoplado) e $F_{\text {esc }}=0.040$ (desacoplado)).

Figura 5.75: Mapeamento das bifurcações locais na região de ressonância fundamental, modelo acoplado e desacoplado, para $\alpha=1.18$, $\varphi=90^{\circ}, \lambda=0.7, \beta=120^{\circ}$ e $\xi_{1}=\xi_{2}=0.01$. Modelo de torre estaiada considerando a influência da rigidez relativa das molas.

Figura 5.76: Diagramas de bifurcação, modelo acoplado e desacoplado, para $\Omega=0.82807, \varphi=90^{\circ}, \alpha=1.18, \lambda=0.7, \beta=120^{\circ}$ e $\xi_{1}=\xi_{2}=0.01$. Modelo de torre estaiada considerando a influência da rigidez relativa das molas.

Figura 5.77: Fronteiras de estabilidade (escape) para $\lambda=0.7, \beta=120^{\circ} \mathrm{e}$ $\xi_{1}=\xi_{2}=0.01$. Modelo de torre estaiada com imperfeição geométrica. 239 Figura 5.78: Variação da carga de escape, $F_{\text {esc }}$, com a direção da excitação, $\varphi$, (gráfico em coordenadas polares) para $\lambda=0.7, \beta=120^{\circ} \mathrm{e}$ $\xi_{1}=\xi_{2}=0.01$. Modelo de torre estaiada com imperfeição geométrica. 240 Figura 5.79: Curvas de ressonância para $F=0.005, \phi=1^{\circ}, \psi=90^{\circ}$, $\lambda=0.7, \beta=120^{\circ}$ e $\xi_{1}=\xi_{2}=0.01$. Modelo de torre estaiada com imperfeição geométrica.

Figura 5.80: Mapeamento das bifurcações locais na região de ressonância fundamental, modelo acoplado, para $\phi=1^{\circ}, \psi=90^{\circ}, \lambda=0.7$, $\beta=120^{\circ}$ e $\xi_{1}=\xi_{2}=0.01$. Modelo de torre estaiada com imperfeição geométrica.

Figura 5.81: Diagramas de bifurcação para $\Omega=0.6967, \varphi=0^{\circ}, \phi=1^{\circ}$, $\psi=90^{\circ}, \lambda=0.7, \beta=120^{\circ}$ e $\xi_{1}=\xi_{2}=0.01$. Modelo de torre estaiada com imperfeição geométrica $\left(F_{\text {esc }}=0.0311\right)$.

Figura 5.82: Seções das bacias de atração nos planos $u_{1} x d u_{1} / d t, u_{2} x d u_{2} / d t$ e $u_{1} x u_{2}$, para $\Omega=0.6967, \varphi=0^{\circ}, \phi=1^{\circ}, \psi=90^{\circ}, \lambda=0.7, \beta=120^{\circ} \mathrm{e}$ $\xi_{1}=\xi_{2}=0.01$. Modelo de torre estaiada com imperfeição geométrica. 242 Figura 5.83: Medida de integridade local da bacia de atração, LIM, modelo acoplado, para $\Omega=0.6967, \varphi=45^{\circ}, \phi=1^{\circ}, \psi=90^{\circ}, \lambda=0.7, \beta=120^{\circ} \mathrm{e}$ $\xi_{1}=\xi_{2}=0.01$. Modelo de torre estaiada com imperfeição geométrica. 
Figura 6.1: Ilustração de aplicação do método de controle proposto por Lenci \& Rega.

Figura 6.2: Variedades e perfil da energia potencial para $\Omega=1 / 3$ e $\lambda=0.9$. Modelo perfeito desacoplado. 268

Figura 6.3: Ilustração de uma órbita heteroclínica. 268

Figura 6.4: Órbita heteroclínica para $\Omega=1 / 3$ e $\lambda=0.9$. Modelo perfeito desacoplado.

Figura 6.5: Mapeamento das bifurcações locais na região de ressonância fundamental para $\lambda=0.9$ e $\xi=0.01$. Modelo perfeito desacoplado. 270 Figura 6.6: Diagramas de bifurcação para $\Omega=0.2465, \lambda=0.9$ e $\xi=0.01$, modelo original e modelo controlado $\left(9 F_{3} / F=-1.115279\right.$ e $\left.v_{30 t i m o}=\pi\right)$. Modelo perfeito desacoplado.

Figura 6.7: Variedades invariantes associadas às fronteiras de estabilidade para $F=0.0528979, \Omega=0.2465, \lambda=0.9$ e $\xi=0.01$, modelo original e modelo controlado $\left(9 F_{3} / F=-1.115279\right.$ e $\left.v_{3 o t i m o}=\pi\right)$. Modelo perfeito desacoplado.

Figura 6.8: Medidas de integridade GIM e IF para $\Omega=0.2465, \lambda=0.9$ e $\xi=0.01$, modelo original e modelo controlado $\left(9 F_{3} / F=-1.115279 \mathrm{e}\right.$ $v_{30 \text { timo }}=\pi$ ). Modelo perfeito desacoplado.

Figura 6.9: Bacias de atração para $\Omega=0.2465, \lambda=0.9$ e $\xi=0.01$, modelo original e modelo controlado $\left(9 F_{3} / F=-1.115279\right.$ e $\left.v_{30 t i m o}=\pi\right)$. Modelo perfeito desacoplado.

Figura 6.10: Variedades e perfil da energia potencial para $u_{10}=1^{\circ}, \Omega=1 / 3$ e $\lambda=0.9$. Modelo desacoplado com imperfeição geométrica.

Figura 6.11: llustração de uma órbita homoclínica.

Figura 6.12: Órbita homoclínica para $u_{10}=1^{\circ}, \Omega=1 / 3$ e $\lambda=0.9$. Modelo com imperfeição geométrica.

Figura 6.13: Mapeamento das bifurcações locais na região de ressonância fundamental para $u_{10}=1^{\circ}, \lambda=0.9$ e $\xi=0.01$. Modelo com imperfeição geométrica desacoplado.

Figura 6.14: Diagramas de bifurcação para $u_{10}=1^{\circ}, \Omega=0.254, \lambda=0.9 \mathrm{e}$ $\xi=0.01$, modelo original e modelo controlado $\left(4 F_{2} / F=1.337189 \mathrm{e}\right.$ 
$\left.v_{2 o t i m o}=\pi\right)$. Modelo com imperfeição geométrica desacoplado.

Figura 6.15: Fronteiras de estabilidade para $F=0.02561, u_{10}=1^{\circ}$, $\Omega=0.254, \lambda=0.9$ e $\xi=0.01$, o modelo original e modelo controlado $\left(4 F_{2} / F=1.337189\right.$ e $\left.v_{2 o t i m o}=\pi\right)$. Modelo com imperfeição geométrica desacoplado.

Figura 6.16: Medidas de integridade GIM e IF para $u_{10}=1^{\circ}, \Omega=0.254$, $\lambda=0.9$ e $\xi=0.01$, modelo original e modelo controlado $\left(4 F_{2} / F=1.337189\right.$

e $\left.v_{2 o t i m o}=\pi\right)$. Modelo com imperfeição geométrica desacoplado. 280

Figura 6.17: Bacias de atração para $u_{10}=1^{\circ}, \Omega=0.254, \lambda=0.9$ e $\xi=0.01$, modelo original e modelo controlado $\left(4 F_{2} / F=1.337189\right.$ e $\left.v_{2 o t i m o}=\pi\right)$.

Modelo com imperfeição geométrica desacoplado.

Figura 6.18: Comparação das medidas de integridade GIM e IF do modelo perfeito (original e controlado) com o modelo imperfeito (original e controlado).

Figura 6.19: Variedades e perfil da energia potencial para $\Omega=0.654653$ e $\lambda=0.7$. Modelo perfeito desacoplado.

Figura 6.20: Órbita homoclínica para $\Omega=0.654653$ e $\lambda=0.7$. Modelo perfeito desacoplado.

Figura 6.21: Mapeamento das bifurcações locais na região de ressonância fundamental para $\lambda=0.7$ e $\xi=0.01$. Modelo perfeito desacoplado.

Figura 6.22: Diagramas de bifurcação para $\Omega=0.5456, \lambda=0.7$ e $\xi=0.01$, modelo original e modelo controlado $\left(4 F_{2} / F=1.160502\right.$ e $\left.v_{2 o t i m o}=\pi\right)$. Modelo perfeito desacoplado.

Figura 6.23: Medidas de integridade GIM e IF para $\Omega=0.5456, \lambda=0.7 \mathrm{e}$ $\xi=0.01$, modelo original e modelo controlado $\left(4 F_{2} / F=1.160502 \mathrm{e}\right.$ $v_{2 o t i m o}=\pi$ ). Modelo perfeito desacoplado.

Figura 6.24: Bacias de atração para $\Omega=0.5456, \lambda=0.7$ e $\xi=0.01$, modelo original e para o modelo controlado $\left(4 F_{2} / F=1.160502\right.$ e $\left.v_{2 o t i m o}=\pi\right)$. Modelo perfeito desacoplado. 


\section{Lista de Tabelas}

Tabela 4.1: Freqüências naturais e modos lineares de vibração. Modelo de Augusti.

Tabela 4.2: Freqüências naturais e modos lineares de vibração. Modelo de torre estaiada.

Tabela 5.1: Freqüências naturais para $\lambda=0.9$. Modelo de Augusti perfeito.

Tabela 5.2: Freqüências naturais para $\lambda=0.9$. Modelo de Augusti considerando a influência da rigidez relativa das molas.

Tabela 5.3: Freqüências naturais para $\lambda=0.9$. Modelo de Augusti com imperfeição geométrica.

Tabela 5.4: Freqüências naturais para $\lambda=0.7$ e $\beta=120^{\circ}$. Modelo de torre estaiada perfeito.

Tabela 5.5: Freqüências naturais para $\alpha<1, \lambda=0.7$ e $\beta=120^{\circ}$. Modelo de torre estaiada considerando a influência da rigidez relativa das molas.

Tabela 5.6: Freqüências naturais para $\alpha>1, \lambda=0.7$ e $\beta=120^{\circ}$. Modelo de torre estaiada considerando a influência da rigidez relativa das molas.

Tabela 5.7: Freqüências naturais para $\lambda=0.7$ e $\beta=120^{\circ}$. Modelo de torre estaiada com imperfeição geométrica.

Tabela 6.1: Resultados numéricos dos problemas de otimização com o aumento do número de super-harmônicos no caso de controle one-side.

Tabela 6.2: Resultados numéricos dos problemas de otimização com o aumento do número de super-harmônicos no caso de controle global. 


\section{Lista de Símbolos}

$C$ c constante igual ou menor que à energia associada aos pontos de sela dos modelos;

$C_{i}$, parâmetros de amortecimento, expressos pelas taxas de amortecimento, $\xi_{i}$;

$D_{b}(t), \quad$ excitação harmônica de base;

DH, vetor que representa a parte não perturbada do sistema;

E, $\quad$ parcela de amortecimento;

F, $\quad$ amplitude da excitação harmônica de base, $F=F_{b} / l$;

$\mathrm{Fcr}^{h}$ valor teórico da interseção homo/heteroclínica para uma excitação harmônica; valor teórico da interseção homo/heteroclínica para uma excitação harmônica com controle (adição de super-harmônicos);

$F_{b}$, magnitude do deslocamento de base;

$F_{i}$, $\quad$ amplitude da excitação dos super-harmônicos de ordem $i$;

$F_{\text {esc }}$, amplitude crítica, carga de escape;

g, aceleração da gravidade;

g, vetor que representa a parte perturbada do sistema;

$G^{\text {hom }} \quad$ controle de uma órbita homoclínica;

$G^{\text {het }}$, controle de uma órbita heteroclínica;

GIM , medida global de integridade;

$H$, Hamiltoniano dos modelos;

$h$, nível de energia adotado, $H=h$.

$h_{j}$, parâmetros de controle;

IF , fator de integridade;

$k_{i}$, constantes de rigidez das molas;

l, $\quad$ comprimento da coluna;

$L$, função de Lagrange;

$L_{p}$, $\quad$ parcela do potencial gravitacional das cargas externas; 
LIM , medida de integridade local;

$m$, massa concentrada na extremidade livre da coluna;

$M(m)$, função de Melnikov;

$P, \quad$ peso da massa concentrada na extremidade livre da coluna, $P=m g$;

$\mathrm{Pcr}_{i}, \quad$ cargas críticas dos modelos;

t, tempo;

$T$, parcela da energia cinética;

$u$, coordenada auxiliar, para desacoplar os modelos;

$u_{i}$, grandezas $u_{i}=\operatorname{sen} \theta_{i}$, que representam os graus de liberdade do modelo de torre estaiada;

parcelas da imperfeição geométrica do modelo de torre estaiada, respectivamente, nas direções dos graus de liberdade $u_{i}$;

$u_{b}(t)$ parcela da excitação harmônica de base na direção $x$;

$u_{D i}$, deslocamentos dinâmicos, deformações devidas ao movimento;

$u_{\text {esti }}$, rotações estáticas;

$u_{h}$, órbita do sistema (homoclínica $-u_{\text {hom }}$ ou heteroclínica - $u_{\text {het }}$ );

$u_{T i}$, rotações totais;

$u_{S i}$, deformações estáticas;

$U$, parcela da energia interna de deformação;

v, coordenada auxiliar, para desacoplar os modelos;

$v_{b}(t), \quad$ parcela da excitação harmônica de base na direção $y$;

$V$, parcela da energia potencial total;

$\alpha$, relação entre as constantes de rigidez, $k_{i}$;

$\alpha_{i}(j), \quad$ termos que simplificam a formulação do controle;

$\beta$, $\quad$ ângulo que define a posição das molas do modelo de torre estaiada;

$\Delta$, $\quad$ deslocamento vertical total de $m$ medido em relação à configuração indeformada da coluna perfeita;

$\Delta_{f}, \quad$ deslocamento vertical da carga na coluna imperfeita;

$\Delta_{0}$, deslocamento vertical de $m$ devido à imperfeição geométrica;

$\Delta_{L i}$, $\quad$ variação de comprimento das molas do modelo de torre estaiada;

$\varepsilon$, $\quad$ parâmetro adimensional que mede a amplitude da perturbação; 


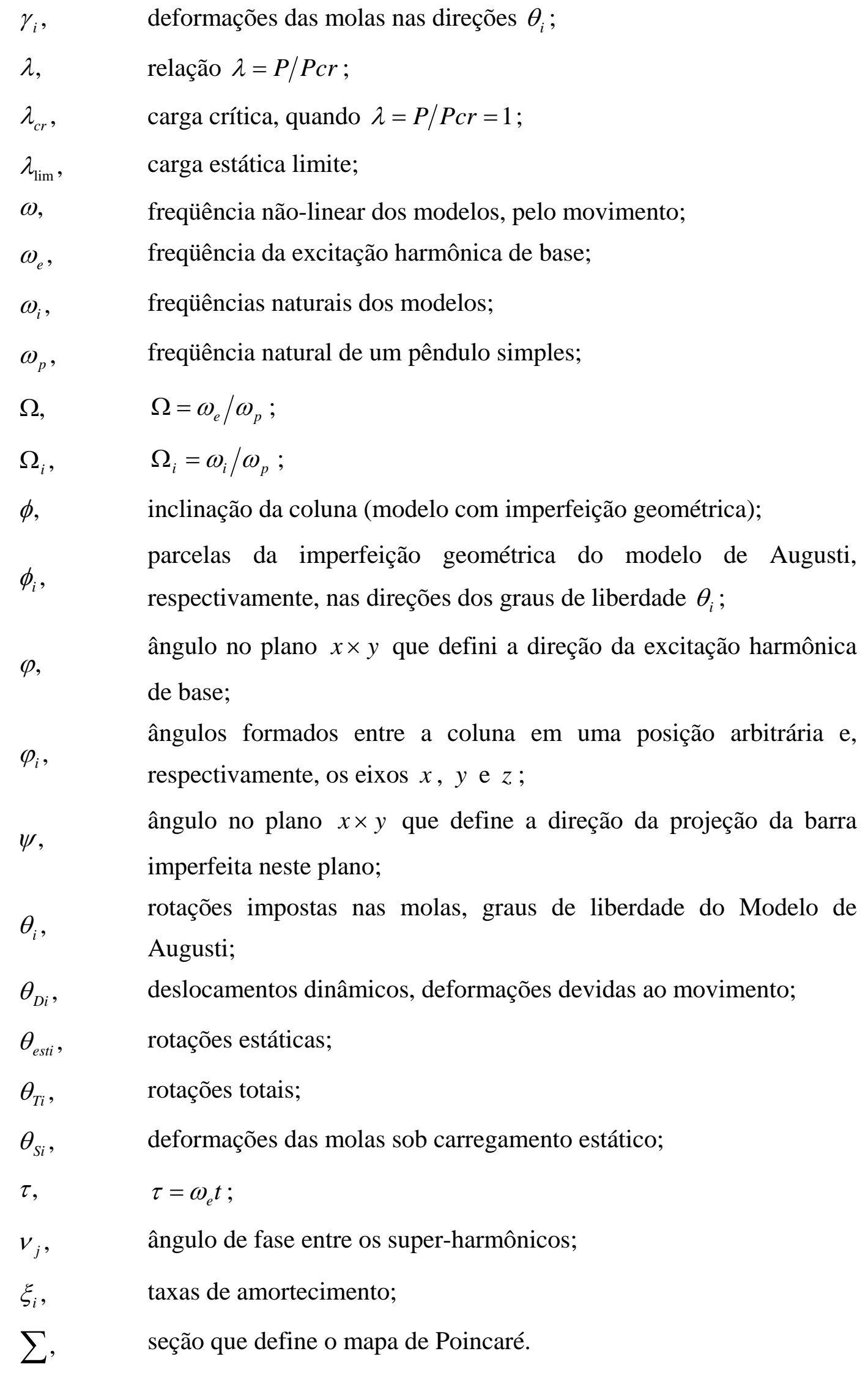

